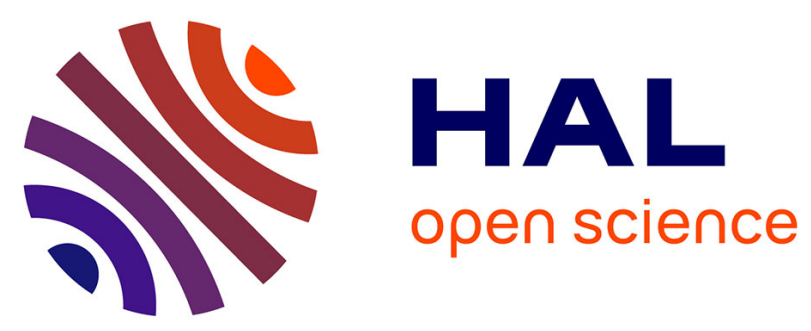

\title{
Impact of on-body channel models on positioning success rate with UWB Wireless Body Area Networks
}

\author{
Arturo Guizar, Claire Goursaud, Bernard Uguen
}

\section{To cite this version:}

Arturo Guizar, Claire Goursaud, Bernard Uguen. Impact of on-body channel models on positioning success rate with UWB Wireless Body Area Networks. EAI International Conference on Body Area Networks (BodyNets), Sep 2015, Sydney, Australia. 10.4108/eai.28-9-2015.2261545 . hal-01246937

\section{HAL Id: hal-01246937 \\ https://hal.science/hal-01246937}

Submitted on 20 Dec 2015

HAL is a multi-disciplinary open access archive for the deposit and dissemination of scientific research documents, whether they are published or not. The documents may come from teaching and research institutions in France or abroad, or from public or private research centers.
L'archive ouverte pluridisciplinaire HAL, est destinée au dépôt et à la diffusion de documents scientifiques de niveau recherche, publiés ou non, émanant des établissements d'enseignement et de recherche français ou étrangers, des laboratoires publics ou privés. 


\section{Impact of on-body channel models on positioning success rate with UWB Wireless Body Area Networks}

\author{
Arturo Guizar \\ University of Lyon \\ INSA-Lyon, CITI-INRIA \\ 69100, Villeurbanne, France \\ arturo.guizar@insa- \\ lyon.fr
}

\author{
Claire Goursaud \\ University of Lyon \\ INSA-Lyon, CITI-INRIA \\ 69100, Villeurbanne, France \\ claire.goursaud@insa- \\ lyon.fr
}

\author{
Bernard Uguen \\ Université Rennes 1 - IETR, \\ F-35042, Rennes, France \\ bernard.uguen@univ- \\ rennes1.fr
}

\begin{abstract}
In this paper, we aim to evaluate the positioning success rate of nodes placed on the body using different scheduling strategies at the Media Access Control (MAC) layer with Ultra Wide Band (UWB) Wireless Body Area Networks (WBAN) and under three different channel models. For this purpose, each node calculates its relative position with the estimation of its distances with the on-body anchors. Accordingly, the distance between two nodes can be estimated with the transmission of three packets, as defined by the '3-Way ranging' protocol (3-WR). However, these transactions can be affected by the WBAN channel leading into a packet loss and therefore positioning errors. In this work, we consider a PHY layer based on Impulse-Radio UWB (IR-UWB) with three different channels: (a) a theoretical path loss channel model based on the on-body CM3 channel (Anechoic chamber), (b) a simulated channel calculated with the PyLayers ray-tracing simulator and (c) experimental traces obtained by measurement. Moreover, we analyze the positioning success rate using three scheduling strategies (Single node localization (P2P), Broadcast Single node localization (P2P-B) and Aggregated \& Broadcast (A\&B)) with a MAC layer based on time division multiple access (TDMA) and under a realistic pedestrian walking scenario. Our results show that the scheduling strategy with A\&B let the nodes to estimate more positions even through channels with slow and fast fading.
\end{abstract}

\section{Categories and Subject Descriptors}

C.2.1 [Network Architecture and Design]: Wireless communication; D.3.3 [Network Architecture and Design]: System design issues, and performance modeling; H.1.2 [Models and Principles]: Miscellaneous

\section{General Terms}

Performance, Measurement, Experimentation

\section{Keywords}

Wireless Body Area Networks, Ultra Wide Band, Localization, Motion Capture, IEEE 802.15.6, Scheduling

\section{INTRODUCTION}

Human movement detection has been extensively studied on the last years because of its importance on healthcare applications i.e. fall detection, assisted navigation and rehabilitation. Among the different techniques, motion capture is possible with WBAN [1]. This technology can collect different data from the human activity. Thus, WBAN can perform the localization with inertial sensors [6] (i.e. accelerometer, gyroscope) or with the estimation of the distances between the nodes embedded on the body. This last is possible with IR-UWB [7], which allows accurate precision and acceptable range with low cost and complexity. Here, the distance can be estimated with the transmission of three packets (a request and two responses) as defined by the 3-WR protocol [4]. Several works dealing with UWB ranging estimation were proposed with the wireless sensor networks (WSN) constraints. This is a first problem because the nodes of a WSN are quasi-static, which is not the same for WBAN where nodes are attached to the body. Therefore, their communication can be affected by the random mobility of nodes.

In our previous works [3], we studied the impact of the mobility on the ranging and positioning estimation. We showed that the 3WR ranging transactions may be affected by the node speed and the packets delays taken by the on-body anchors to send the 3-WR responses. In particular, we found that the delay taken for the first response $(\Delta t 1)$ has more impact on the ranging estimation. By that time, we considered a perfect channel in order to quantify only the impact of mobility. However, in real scenarios the channel can produce packets loss. In fact, each mobile node needs to perform the ranging estimation with at least 4 anchors (e.g. time difference of arrival (TDOA) [8]) to achieve a three-dimensional positioning. If one of the 3-WR packets is loss during the current transaction, this means that the node will not be able to evaluate the distance with an anchor and therefore, its position. We propose to handle this problem in a more lightweight way: by using a dedicated MAC layer based on TDMA with three different scheduling strategies. In this paper, we consider the case of a WBAN trying to find their relative positions with on-body anchors composing a local coordinated system (LCS). For this, we implement three channel models: (a) an on-body path loss based on the CM3 UWB channel [9], (b) a simulated channel model calculated by ray tracing with the simulator PyLayers [5] and (c) an experimental channel obtained from a realistic measurement campaign [2]. Then, we evaluate the positioning success rate using the three different MAC schemes. The results show that $A \& B$ is the best mechanism to achieve the positioning of nodes, even with a channel with high packet loss rate. 
The reminder of this study is organized as follows: section 2 gives an overview of the system model such as the topology, the mobility scenario and the localization basics. Section 3 describes the path loss model for the on-body, the simulated channel calculation and the experimental channel properties, along with the MAC strategies used for the 3-WR transactions. Section 4 provides full detail on our results and scenarios. Finally, Section 5 draws a short discussion of our conclusions and perspectives.

\section{SYSTEM MODEL}

\subsection{Network Topology}

Let us consider a WBAN in full-mesh connectivity where all nodes can communicate pair-to-pair. We define two types of sensors: the on-body anchor nodes that have perfect knowledge of their relative and absolute positions at any time and the on-body mobile nodes who want to estimate their relative positions. These sensors communicate with a physical layer based on IR-UWB pulses. Accordingly, each on-body node $i$ estimates its distance $\left(\hat{d}_{i j}(t)\right)$ with the on-body anchor $j$. Then, it estimates its position $\hat{P}_{i}(t)$ with all the distances estimated.

\subsection{Ranging estimation with 2-WR and 3-WR}

To achieve the localization, the 3-Way ranging protocol [4] estimates the distance between the anchor and mobile nodes with the time of arrival (ToA) of three different packets (Fig. 1):

- First, the node $i$ send a Request $\left(Q_{i j}\right)$ to the anchor $j$.

- Then, the anchor $j$ answers with a Response packet $\left(R 1_{i j}\right)$. At this stage, it is possible to calculate the distance with the Time of Flight (ToF) estimated with the 2-WR

- Finally, the anchor $j$ sends a second Response $\left(R 2_{i j}\right)$ to complete the 3-WR transaction and compensate the clock drift between the nodes.

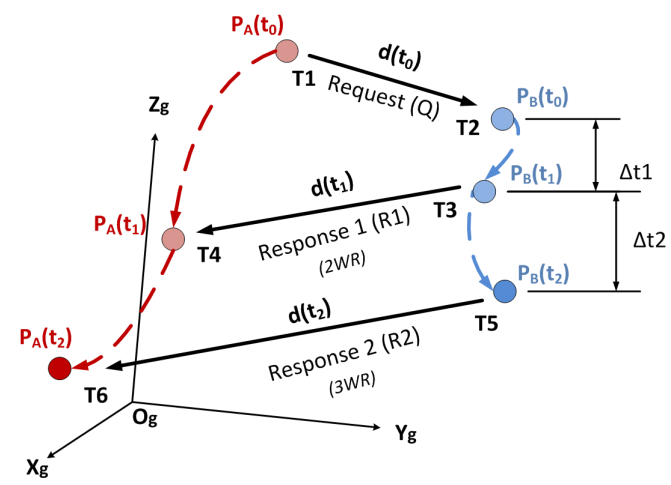

Figure 1: 3-WR protocol applied with mobile nodes

During these transactions, the on-body nodes collect the different timers (ToA) of the 3-WR packets transmission and reception. Thus, the distance $\hat{d}_{i j}(t)$ is evaluated as follows:

$$
\hat{d}_{i j}(t)=\frac{1}{2} c\left[\left(\left(T_{4}-T_{1}\right)-\Delta t 1\right)-\left(\left(T_{6}-T_{4}\right)-\Delta t 2\right)\right]
$$

where $c$ represents the speed of pulses transmission (i.e. $c=3 *$ $\left.10^{8} \mathrm{~m} / \mathrm{s}\right)$ and $\Delta t 1$ (resp. $\left.\Delta t 2\right)$ is the delay between $\left(T_{3}-T_{2}\right)$ (resp. $\left(T_{5}-T_{3}\right)$ ).

\subsection{Mobility Model}

In this study, we aim to evaluate the performance of an UWB WBAN system in a daily context, such as the walking scenario. The mobility model was obtained during the measurement campaign related to the CORMORAN project at the M2S laboratory, ENS Cachan, France in June 2014 [2]. We deployed 16 cameras (Vicon) based on infrared technology at a rate of $100 \mathrm{~Hz}$. Thus, the motion capture was performed in a confined space of $10 \times 6 \mathrm{~m}^{2}$.

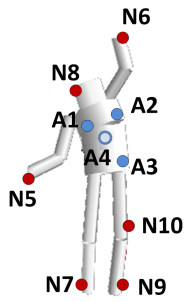

(a)

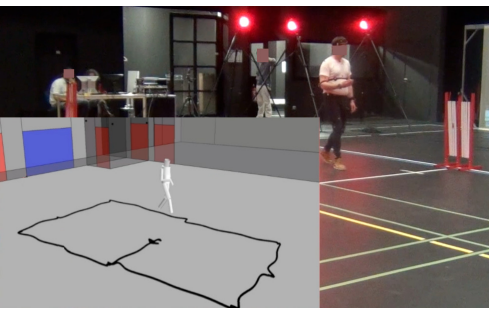

(b)
Figure 2: (a) Multi-cylinder body reconstruction. (b) Pedestrian walking scenario and representation with PyLayers.

Accordingly, we consider a pedestrian walking scenario of $100 \mathrm{~s}$. The person starts in the middle of the scene performing a $360^{\circ}$ rotation with pause of 5 seconds every $90^{\circ}$, then he starts moving at moderate speed along a rectangular trajectory centered on the starting point. The WBAN is composed by 4 anchors and 6 mobile nodes, as shown in Figure 2(a). The on-body anchors are positioned on: the right chest $\left(A_{1}\right)$, the left chest $\left(A_{2}\right)$, the left hip $\left(A_{3}\right)$ and the back $\left(A_{4}\right)$. The mobile nodes are located on the right $\operatorname{arm}\left(N_{5}\right)$, the left arm $\left(N_{6}\right)$, the right foot $\left(N_{7}\right)$, the head $\left(N_{8}\right)$, the left foot $\left(N_{9}\right)$ and the left knee $\left(N_{10}\right)$.

\section{METHODOLOGY}

\subsection{Scheduling MAC Protocols}

We present different MAC strategies to evaluate the positioning success rate. We consider that a positioning is achieved if a node $i$ is able to complete the 3 -WR transactions $(\kappa)$ with the M anchors on the network (at least 4 for positioning in 3D [8]). Accordingly, we perform the positioning with a MAC frame composed by enough slots to deliver the 3 -WR packets with all the anchors. Moreover, we use 3 different MAC strategies (Figure 3) based on TDMA (beacon-enabled and synchronized), as proposed in [3]:

- Single node localization (P2P) where each node $i$ send the request packets $Q_{i j}$ to the anchors in order. Then, each anchor answers with $R 1_{j i}$ and $R 2_{j i}$ successively in singlelinks to the nodes.

- Broadcasted single node localization (P2P-B) where each node $i$ intend to send the requests $Q_{i}$ to the anchors in broadcast. Thereafter, each anchor answers with $R 1_{j i}$ and $R 2_{j i}$ successively in single-links.

- Aggregated and Broadcast (A\&B) [4] where nodes send the requests $Q_{i}$ in broadcast. Then, each anchor $j$ gathers the ToA of each request and sends a response $\left(R 1_{j}\right)$ with all the aggregated timers, followed by the response $\left(R 2_{j}\right)$.

\subsection{On-body channel models}

In this work, we evaluate the positioning success under a realistic mobility scenario and three different channel models. We mimic 

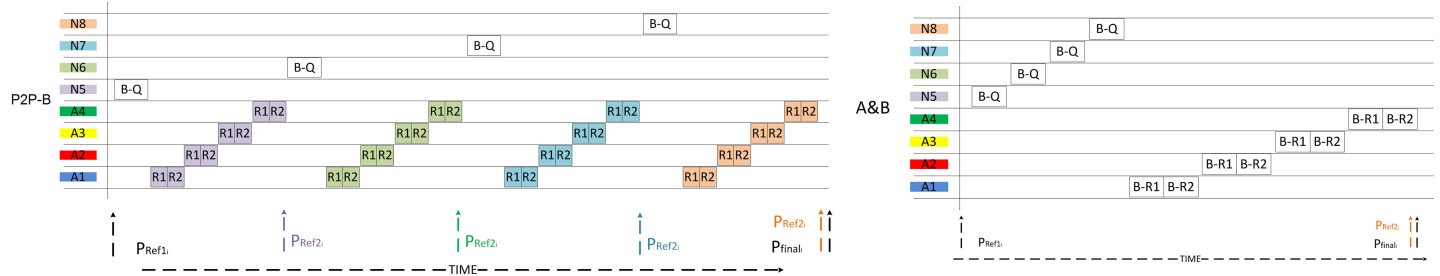

Figure 3: P2P-B and A\&B scheduling strategies

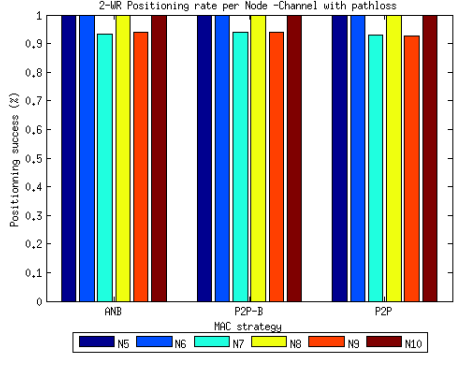

(a) Positioning success rate with $2-\mathrm{WR}$

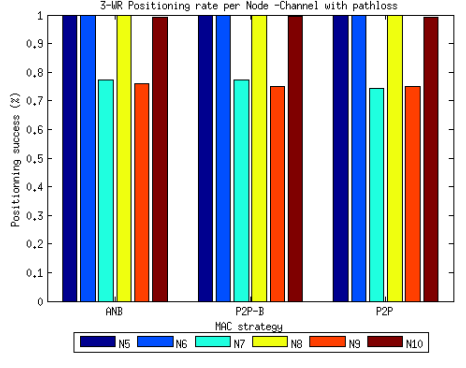

(b) Positioning success rate with $3-\mathrm{WR}$

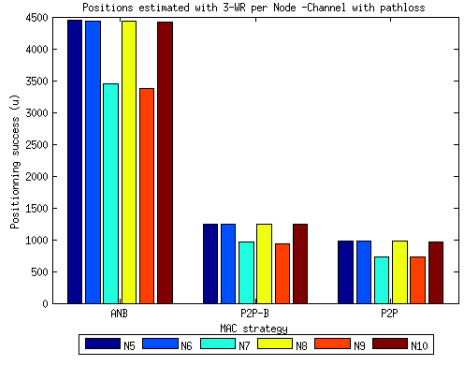

(c) Positions estimated with 3-WR

Figure 4: Comparison of positioning success between the MAC strategies (A\&B, P2P-B and P2P) with the path loss channel

the motion capture process where mobile try to find their positions in the LCS with the anchors nodes embedded on the body. For all the channel models, we define the Packet Error Rate $\left(P E R_{\kappa}\right)$ as the probability to loose a 3 -WR packet $\kappa$ when it is received with a Rx power $\left(P_{R x}\right)$ lower than a certain Rx threshold $(\rho)$ :

$$
P E R_{\kappa}=\mathbb{P}\left(P_{R x}\left(d_{i j}\right) \leq \rho\right)
$$

Path loss model. This theoretical channel is based on the scenario CM3 (3.1-10.6 GHz) as described in [9]:

$$
P L_{\text {on-body }}(d)[d B]=a * \log _{10}(d)+b+N
$$

where $\mathrm{a}$ and $\mathrm{b}$ are linear fitting parameters of the model, $\mathrm{N}$ is a Normal distributed variable with zero mean and standard deviation $\sigma_{N}$, and $d$ is the distance (in $\mathrm{mm}$ ) between the On-body nodes. In our study we use the parameters defined for the Anechoic Chamber which fits more with our mobility scenario (Section 2.3): $a=34.1$, $b=-31.4$ and $\sigma_{N}=4.85$. The power of a received packet is calculated in function of the power of transmission $\left(P_{T x}\right)$ and the gains of the transceiver $\left(G_{T x}\right)$ and receiver $\left(G_{R x}\right)$ as follows $P_{R x}=P_{T x}+G_{T x}+G_{R x}-P L(d)$.

Simulated channel model. In this model, we use a full ray tracing simulation of the radio links in large band calculated by PyLayers. We consider an antenna model [5] mounted within the mobility model to consider the position and orientation of the simulated devices. Note that this channel presents a selective fading which can provoke important packet loss rate.

Experimental channel model. This channel was obtained by real measurements during the CORMORAN campaing [2] using $\mathrm{HiKoB}$ FOX sensors with a PHY layer based on the IEEE $802.15 .4(2.45 \mathrm{GHz}$ ISM band) and a MAC layer based on TDMA. Thus, the experimental traces represents the average Received Signal Strength Indicator (RSSI) from the last 8 modulated symbols. In particular, this channel is characterized by the slow and fast fading in full-mesh.

\section{PERFORMANCE EVALUATION}

\subsection{Simulation tools}

We use the discret-event simulator WSNet to implement the different channel models, suitable for the evaluation of the positioning success rate. In particular, we implemented a MAC a protocol based on TDMA with the strategies P2P, P2P-B and A\&B, as detailed in Section 3.1. The duration of the MAC frame is designed according to the standard IEEE802.15.6 UWB PHY layer on default mode (OOK modulation and $0.4875 \mathrm{Mb} / \mathrm{s}$ ). For the path loss model, the transmitter power is fixed to $P_{T x}=-10 \mathrm{dBm}$ and the antenna gain to $G_{T x}=G_{R x}=0 d B$. Finally, we exploit the traces of the walking scenario acquired during the CORMORAN project, as described in Section 2.3. For all the channel, we fix the sensitivity threshold to $\rho=-91 \mathrm{dBm}$ (w.r.t the standard default mode).

\subsection{On-body positioning rate related to the sen- sitivity threshold}

Figures 4(a), 5(a) and 6(a) (resp. Figs. 4(b), 5(b) and 6(b)) represent the positioning success rate when distances are estimated with 2-WR (resp. 3-WR) with the different channel models. We observe that 2-WR compensates the 3-WR loss and increases the positioning success rate, specially with the simulated channel. However, this cannot be a final conclusion because it is necessary to evaluate the ranging error (clock drift) with 2-WR. Moreover, the results show that $N 7$ and $N 9$ have the lower positioning success rate with all the channels and MAC strategies. This is because the nodes placed on the legs are more affected by the slow/fast fading and path loss effects. When comparing results from path loss and experimental models, they seem to be similar but they are not the same, this is because of the sensitivity threshold. If we analyze the evolution of links Rx power (not presented here) with both models, we observe that almost all the experimental slow/fast fading and the path loss decays are over the Rx threshold. On the contrary, the slow/fast fading from simulated channel are below the sensitivity threshold. By changing this threshold, experimental results would be more affected than the path loss results. Finally, we note that the 


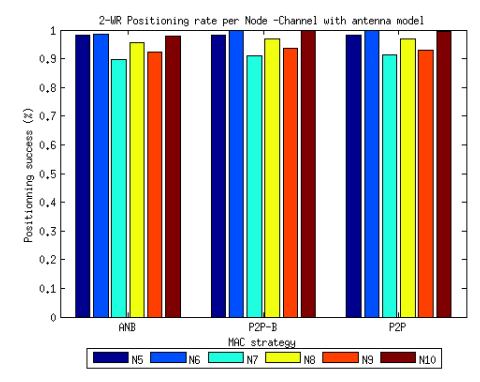

(a) Positioning success rate with 2-WR

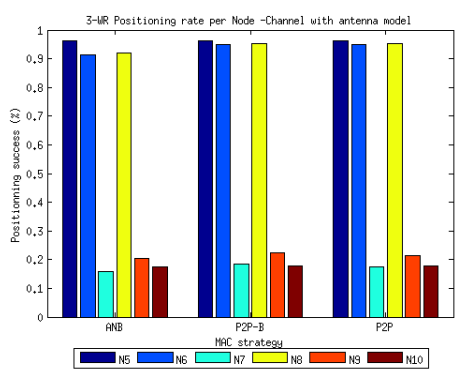

(b) Positioning success rate with 3-WR

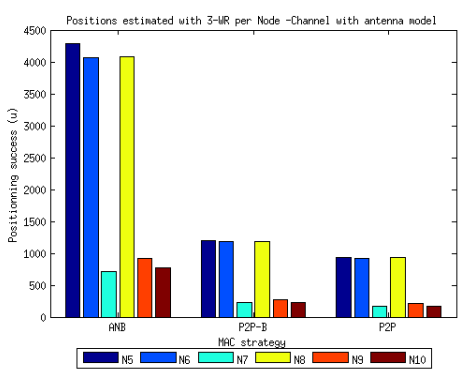

(c) Positions estimated with 3-WR

Figure 5: Comparison of positioning success between the MAC strategies (A\&B, P2P-B and P2P) with the simulated channel

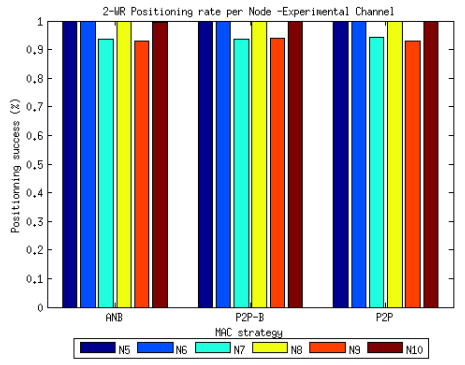

(a) Positioning success rate with $2-\mathrm{WR}$

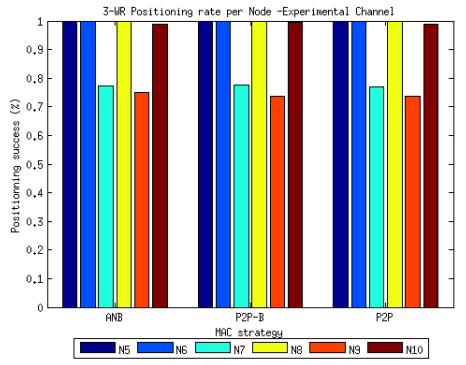

(b) Positioning success rate with 3-WR

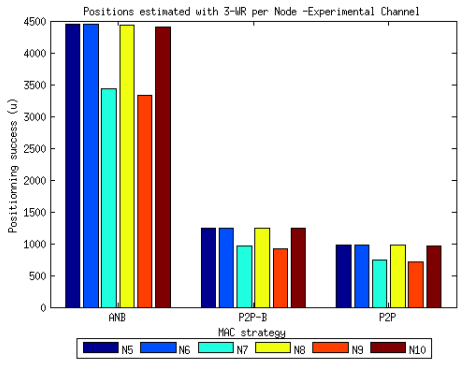

(c) Positions estimated with 3-WR

Figure 6: Comparison of positioning success between the MAC strategies (A\&B, P2P-B and P2P) with the experimental channel

different MAC strategies have similar positioning success rate, but when we compare with Figures 4(c), 5(c) and 6(c)), we observe that $\mathrm{A} \& \mathrm{~B}$ is the best choice because it let us estimate more positions.

\section{CONCLUSION}

We have presented a first analysis of the impact of different channel models on the positioning success rate with WBAN using IRUWB. We consider a scenario where on-body nodes communicate with on-body anchors to perform individual positioning. For this purpose, we use the CM3 path loss model as defined by the standard IEEE 802.15.6, a simulated channel calculated with ray-tracing by PyLayers and an experimental channel obtained by measurement. We show that the positioning success rate is affected by all the channel models with a fixed sensitivity threshold. Moreover, we found that $\mathrm{A} \& \mathrm{~B}$ is the best choice because it let us estimate more positions. Future work will consist in the study of body-to-body and off-body localization under different channel situations, different sensitivity threshold and realistic mobility models.

\section{ACKNOWLEDGMENT}

This work has been carried out in the frame of the CORMORAN project, which is funded by the French National Research Agency (ANR) under the contract number ANR-11-INFR-010.

\section{REFERENCES}

[1] R. Cavallari, F. Martelli, R. Rosini, C. Buratti, and $\mathrm{R}$. Verdone. A survey on wireless body area networks: Technologies and design challenges. IEEE Communications Surveys \& Tutorials, 16(3):1635-1657, 2014.

[2] B. Denis, N. Amiot, B. Uguen, A. Guizar, C. Goursaud, A. Ouni, and C. Chaudet. Qualitative Analysis of RSSI
Behavior in Cooperative Wireless Body Area Networks for Mobility Detection and Navigation Applications. In 21st IEEE International Conference on Electronics Circuits and Systems, Marseille, France, Dec. 2014.

[3] A. Guizar, A. Ouni, C. Goursaud, C. Chaudet, and J. Gorce. Quantifying the impact of scheduling and mobility on IR-UWB localization in body area networks. In 12th IEEE International Conference on Body Sensor Networks, MIT, Cambridge, USA, 2015.

[4] D. Macagnano, G. Destino, F. Esposito, and G. Abreu. MAC performances for localization and tracking in wireless sensor networks. 2007 4th Workshop on Positioning, Navigation and Communication.

[5] M. Mhedhbi, N. Amiot, S. Avrillon, and B. Uguen. Human Body Perturbed Antenna Integration In Indoor Propagation Simulator. In Journées scientifiques 2014 de l'URSI France, Paris, France, Mar. 2014.

[6] T. Sakaguchi, T. Kanamori, H. Katayose, K. Sato, and S. Inokuchi. Human motion capture by integrating gyroscopes and accelerometers. 1996 IEEE/SICE/RSJ International Conference on Multisensor Fusion and Integration for Intelligent Systems, 1996.

[7] H. Soganci, S. Gezici, and H. Poor. Accurate positioning in ultra-wideband systems. IEEE Wireless Communications, 18(2):19-27, April 2011.

[8] Z. Xiao, Y. Hei, Q. Yu, and K. Yi. A survey on impulse-radio UWB localization. Sci. China Inf. Sci., 53(7):1322-1335, 2010.

[9] K. Y. Yazdandoost and K. Sayrafian-Pour. Channel model for body area network (ban), 2009. 